\title{
Ablation for paroxysmal atrial fibrillation-real-life results from a middle-volume electrophysiology laboratory
}

\author{
Piotr Kulakowski ${ }^{1} \cdot$ Agnieszka Sikorska $^{1} \cdot$ Roman Piotrowski $^{1}$ (D) $\cdot$ Tomasz Kryński $^{1} \cdot$ Jakub Baran $^{1}$
}

Received: 6 September 2020 / Accepted: 28 December 2020 / Published online: 9 January 2021

(C) The Author(s) 2021

\begin{abstract}
Introduction A significant improvement in safety and efficacy of ablation for paroxysmal atrial fibrillation (PAF) has been reported by experienced centers over recent years; however, data from real-life surveys and smaller electrophysiology (EP) laboratories have been less optimistic.

Aim To asses efficacy of ablation for PAF in a middle-volume EP center over last years.

Methods Retrospective analysis of 1 year efficacy and safety of ablation for PAF in three cohorts of patients treated between 2011 and 2014 (period I), 2015-2017 (period II), and 2018-2019 (period III).

Results Of 234 patients (mean age $57 \pm 9$ years, 165 males), 81 (35\%) were treated in period I, 84 (36\%) in period II, and 69 (29\%) in period III. The overall efficacy of ablation during all analyzed periods was $67 \%$. The overall efficacy of ablation increased over time - from $56 \%$ in period I to $68 \%$ in period II and $81 \%$ in period III. Significant improvement was achieved using radiofrequency ablation (RF) (53\% in period I vs $82 \%$ in period III, and $55 \%$ in period II vs $82 \%$ in period III, $p=0.003$ and 0.0012 , respectively) whereas positive trend in the improvement of cryoballoon efficacy was NS. The rate of peri-procedural complications was $9 \%$ and it did not change significantly over time.

Conclusions This real-life observational study from a medium volume EP center shows that progress in PAF ablation, especially $\mathrm{RF}$, reported by highly-skilled centers, can be reproduced in real life by less experienced operators.
\end{abstract}

Keywords Atrial fibrillation · Ablation · Efficacy

\section{Introduction}

Ablation for paroxysmal atrial fibrillation (PAF) has been introduced into clinical practice over 20 years ago [1]. Pulmonary vein isolation (PVI) is a cornerstone of ablation in PAF [1]. A significant improvement in safety and efficacy of PVI has been documented by numerous scientific reports, generated by top-skilled and very experienced centers $[2,3]$; however, data from real-life surveys have been less optimistic [4]. Operator's growing experience, advances in technology, better understanding of AF mechanisms, and better patient's selection have probably improved ablation success rate in

Roman Piotrowski

rpiotrow@op.pl

1 Electrophysiology Laboratory, Department of Cardiology, Centre for Postgraduate Medical Education, Grochowski Hospital,

Grenadierow 51/59, 04-073 Warsaw, Poland everyday life in recent years; however, data are limited [3]. In addition, there was some criticism in the past that results of $\mathrm{AF}$ ablation were sub-optimal and not cost-effective [5]. In this context, data confirming significant improvement in AF ablation efficacy and safety in real world would be welcomed.

Additionally, in many countries, the vast majority of electrophysiology (EP) laboratories perform less than $50 \mathrm{AF}$ ablations per year (in the USA even less than 20) [6]. A real-life success rates vary between 45 and $74 \%[4,7]$ whereas major complication rate is increased in smaller centers, reaching $9 \%$ [6]. Therefore, it would be interesting to know whether novel technology and new ablation techniques improved ablation success rate in a real-life scenario in a typical middlevolume EP center.

\section{Aim}

To asses efficacy of ablation for PAF in a middle-volume EP center over last years and to identify factors associated with success rate improvement. 


\section{Methods}

Study group Between June 2011 and October 2019, 775 ablation procedures for $\mathrm{AF}$ and 83 ablations for post-ablation left atrial tachycardia/flutter were performed in our center. Of these, 234 patients (mean age $57 \pm 9$ years, 165 males) were included in three prospective studies conducted in our laboratory $[8,9]$ in which the follow-up methodology was identical (NCT03811639, NCT03877913), enabling comparisons between groups of patients treated in various periods. All patients had to have PAF only and no previous AF ablation.

Three time-intervals were defined. The first time-period (period I) was set between June 2011 and February 2014. At that time, the first study [8] was conducted and the Navistar Thermocool SF (Biosence Webster, USA) ablation catheter with the CARTO 3 System (Biosence Webster, USA) was used for RF ablations whereas for cryoablation, the first generation of cryoballoon (CB) (Arctic Front 2AF281, Medtronic, USA) was used. The second time-period (period II) was set between October 2015 and December 2017 when the ABLANSAF study (NCT03811639) was conducted (9). At that time, the Thermocool SmartTouch catheter, CARTO 3 system with Visitag software (Biosence Webster, USA), was used for RF ablation and second generation of $\mathrm{CB}$ (Arctic Front 2AF283, Medtronic, USA) became available. The last time-period (period III) was set between January 2018 and October 2019 when the ABLANSAF study was ended and a new ongoing study AGNES (NCT03877913) was initiated. At that period, the Thermocool SmartTouch catheter, CARTO 3 system, ablation index, and CLOSE protocol were used and the use of second generation of $\mathrm{CB}$ was continued.

Ablation methodology During all analyzed periods, RF ablation was performed using double transseptal puncture for ablation catheter and circular mapping catheter. Changes in RF ablation methodology over time are described below. The CB ablation was performed in standard manner, using a long $8.5 \mathrm{~F}$ sheath and Brockenburgh needle for transseptal puncture, then exchanging it for a flexible 15F sheath (FlexCath Advance, Medtronic, USA) and inserting a balloon with diagnostic Achieve electrode (Medtronic, USA). One important difference between patients treated with RF vs $\mathrm{CB}$ was the presence of the common trunk of left PV. When this type of anatomy was detected on the pre-procedural cardiac computed tomography $(\mathrm{CT})$ or intra-procedural intracardiac echocardiography (ICE), we used RF.

Period I RF ablation was performed with catheters without contact force sensing and the goal of ablation was to achieve PVI not paying too much attention to the quality and contiguity of RF applications which were manually marked on the CARTO map. We used power settings of $20-25 \mathrm{~W}$ at posterior wall and $30-35 \mathrm{~W}$ at anterior wall. All patients had pre- procedural computed tomography (CT) for left atrium (LA) imaging and subsequent merging into the CARTO system. At that time, ICE was used occasionally and only to guide transseptal puncture.

For $\mathrm{CB}$, the first generation of balloons was used, the freezes had to be of 240-300 s duration, and two applications per one PV were performed. The Achieve catheter and pacing from PV were used to confirm PVI, and this approach did not change throughout all three analyzed periods. The nadir temperature achieved had to be less than $-36^{\circ} \mathrm{C}$ and complete PV occlusion was attempted in each case.

Period II At this time-period, RF ablation changed significantly due to advent of contact force sensing ablation electrodes and further improvements in the CARTO system such as automatic tagging of ablation points (Visitag software). More attention was paid to the quality and contiguity of RF lesions; however, in case of durable PVI, leaving the gaps in the ablation line was still acceptable. The power settings remained unchanged. Also, catheter dragging instead of separate pointby-point technique was sometimes used by some operators. Pre-procedural LA imaging was mixed and consisted mainly of CT or rotational angiography which were subsequently merged with LA CARTO map. The ICE was used routinely for guiding transseptal puncture and for guiding ablation in some cases.

As far as CB was concerned, a new generation of balloons was introduced and more attention was paid to the quality of freezes, allowing for single application per vein and of shorter duration when PVI was achieved quickly $(<100 \mathrm{~s})$ during first freeze.

Period III At that time, further improvements in RF methodology led to the implementation of the ablation index and CLOSE protocol into clinical practice. The RF lesions had to be of predefined quality (ablation index $350-400$ at posterior wall and 450-500 at anterior wall) and closely spaced. The power settings remained unchanged; however, there was a clear end-point of each application - predefined ablation index. From the beginning of the use of ablation index, we decided to tag 2-mm dots instead of $3 \mathrm{~mm}$ advocated in the CLOSE protocol because we believed that such an approach further reduced the possibility of leaving gaps in the ablation line. We stopped to perform pre-procedural $\mathrm{CT}$ or rotational angiography and merging them with the CARTO map. Instead, we fully capitalized on our growing experience with ICE and used this modality to help in constructing CARTO map with ablation catheter and/or circular catheter. ICE was also used to guide ablation.

As far as CB is concerned, we started to use routinely time to PVI and thawing time as additional parameters to decide how long and how many freezes were needed to obtain PVI. Again, the use of ICE was expanded and it served for 
confirming complete PV occlusion in case of doubtful fluoroscopy images and to accurately position the Achieve catheter (not too deep in PV and not to proximal in LA).

Other procedural details All ablations were performed in patients in mild sedation with midazolam and fentanyl. The ACT was kept $>350 \mathrm{~s}$ during all analyzed periods. During period I, anticoagulants were stopped before the procedure and the patients were switched to low molecular weight heparin whereas in periods II and III, vitamin $\mathrm{K}$ antagonists were continued, preferably at the INR level of 2.0-3.0 whereas in those taking non-vitamin $\mathrm{K}$ anticoagulants, only the morning dose (directly before the procedure) was omitted and restarted $4 \mathrm{~h}$ after ablation. No protamine at the end of ablation was given. In period I, manual compression followed by bandage compression for $6 \mathrm{~h}$ was used whereas in periods II and III sites of punctures were sutured using a figure-of-eight suture and slight compression was instituted for approximately $6 \mathrm{~h}$. The USG-guided vascular access has been used in our center since January 2017.

Follow-up All 234 patients were followed for 1 year in our outpatient clinic. They attended visits 3, 6, and 12 months after ablation during which history taking, physical examination and ECG were performed. All patients had 7-day Holter ECG recorded at these time-points. Ablation failure was defined as any recurrences of symptomatic AF or atrial tachycardia or any episode of $\mathrm{AF}>30$ s recorded during Holter ECG monitoring. If a patient was on antiarrhythmic drug prior to ablation, the drug was continued in the same dose for 3 months after the procedure and attending physicians were encouraged to stop antiarrhythmic medication at that point. Any increase in the dose of previously administered antiarrhythmic drug or introduction of new antiarrhythmic drug was regarded as ablation failure.

Statistical analysis The results are presented as mean $\pm \mathrm{SD}$ or numbers and percentages. Quantitative data were compared using Student $t$ test or ANOVA test whereas qualitative variables, using chi-square test with Yates' correction or Fisher exact test. A $p$ value $<0.05$ was considered significant.

\section{Results}

Of 234 patients, 81 (35\%) were treated in period I, $84(36 \%)$ in period II, and 69 (29\%) in period III. Demographic and clinical characteristics of analyzed three groups of patients are compared in Table 1. There were no significant differences between the analyzed subgroups.

Overall efficacy The overall efficacy of ablation during all analyzed periods was 67\% (158 patients). Of 123 patients treated with RF ablation, 79 (64\%) were successfully treated compared with 79 (71\%) of 111 patients who underwent CB (NS). The overall efficacy of ablation increased over timefrom $56 \%$ in period I to $68 \%$ in period II and $81 \%$ in period III (Fig. 1). Of 158 effectively treated patients, 143 (91\%) were off antiarrhythmic medication whereas the remaining $15(9 \%)$ patients remained on unchanged doses of antiarrhythmic drugs.

Additional extra-PV ablation was performed in 4 patients from the RF arm (roof line in two, ablation of trigger in coronary sinus and fractionated potentials in one each). One patient treated with $\mathrm{CB}$ had RF touch-up application to finalize isolation of RIPV.

RF ablation vs $C B$ over time Comparison of success rate of $R F$ ablation vs $\mathrm{CB}$ in analyzed time-periods is shown in Fig. 2. There was a constant increase in ablation efficacy over time using $\mathrm{RF}$ technique whereas improvement in $\mathrm{CB}$ was stagnant from period II onwards. Significant improvement was achieved using RF ablation (53\% in period I vs $82 \%$ in period III, and $55 \%$ in period II vs $82 \%$ in period III, $p=0.003$ and 0.012 , respectively), whereas trend in improvement of $\mathrm{CB}$ efficacy was NS.

There were significant differences in the proportion of RF vs CB usage between period II vs periods I and III ( 49 vs 32 in period I, 29 vs 55 in period II, and 45 vs 24 in period III, respectively; $p=0.0008$ for period I vs II, $p=\mathrm{NS}$ for period I vs III, and $p=0.0002$ for period II vs III).

Changes in procedural details in RF group and CB group are shown in Tables 2, 3, and 4. There was a significant decrease in the fluoroscopy time, both using RF and CB whereas the total duration of procedure significantly shortened only in the CB group. The number of isolated PV increased over time using both techniques and reached all $4 \mathrm{PV}$ in the last analyzed period. Analysis of RF details showed that total duration of RF time decreased in period II compared with period I and again increased during period III. The ablation count which depicts the number of RF applications showed similar changes with a marked increase during period III.

Using $\mathrm{CB}$, isolation of all $4 \mathrm{PV}$ was achieved using significantly lower number of freezes and shorter total freezing time per vein in periods III and II compared with period I. The value of nadir temperature slightly but significantly decreased in the left PV.

The usage of ICE for performing transseptal puncture and guiding ablation significantly increased over time both in the $\mathrm{RF}$ and $\mathrm{CB}$ arms.

Complications The rate of peri-procedural complications was 9\%. There were no deaths (neither in-hospital or during follow-up). Also, no atrio-esophageal fistula or persistent phrenic nerve paralysis was noted. The most common were local complications with arterio-venous fistula being the most frequent. 
Table 1 Comparison of demographic and clinical parameters

\begin{tabular}{lllll}
\hline Parameter & Period I $N=81$ & Period II $N=84$ & Period III $N=69$ & $p$ value \\
\hline Age (years) & $57 \pm 10$ & $56 \pm 10$ & $59 \pm 11$ & NS \\
Male gender (\%) & $58(72 \%)$ & $56(67 \%)$ & $51(74 \%)$ & NS \\
BMI & $29.8 \pm 4$ & $28.4 \pm 4$ & $28.1 \pm 4$ & NS \\
$\mathrm{CHA}_{2} \mathrm{DS}_{2}$ Vasc & $1.14 \pm 1.2$ & $1.2 \pm 1.2$ & $1.6 \pm 1.3$ & $\mathrm{NS}$ \\
HAS-BLED & $0.17 \pm 0.39$ & $0.52 \pm 0.63$ & $0.54 \pm 0.72$ & $\mathrm{NS}$ \\
LA size (mm) & $38.7 \pm 4.0$ & $37.6 \pm 4.5$ & $38.0 \pm 4.8$ & $\mathrm{NS}$ \\
LVEF (\%) & $66 \pm 6$ & $59.9 \pm 5$ & $59.3 \pm 4$ & $\mathrm{NS}$ \\
Duration of AF history (years) & $6.6 \pm 5.2$ & $5.6 \pm 3.8$ & $4.6 \pm 3.0$ & $\mathrm{NS}$ \\
\hline
\end{tabular}

$B M I$, body mass index; $L A$, left atrial; $L V E F$, left ventricular ejection fraction
The rate of local complications did not significantly change after introduction of USG-guided vascular access (7/135 (5\%) vs $5 / 99(5 \%)$, NS) and tended to be higher in the RF than in the CB group (8/123 (7\%) vs 4/111 (4\%), NS). Two patients who suffered from TIA or stroke and a patient with cardiac tamponade (perforation of the right atrial appendage with a diagnostic electrode) recovered completely.

\section{Discussion}

\subsection{Technological progress that increases ablation efficacy}

During the analyzed period, a significant improvement in RF technology was introduced. They mainly included the use of contact force catheters and better control of the quality of RF lesions using the ablation index and CLOSE protocol [2]. These changes resulted in improvement in RF ablation efficacy, reported by experienced centers. We also observed a marked increase in efficacy of our procedures which shows that these new developments also work in real life in a middlevolume center. The only difference between our approach and that described in the original CLOSE protocol is that we used slightly lower values of ablation index; however, we used smaller dots representing RF applications on the CARTO map ( $2 \mathrm{~mm}$ instead of $3 \mathrm{~mm}$ ). We believe that the use of smaller dots results in more dense ablation line around PV because more RF application have to be delivered without leaving gaps between RF applications. It is also encouraging that we did not see any excess in such complications as cardiac tamponade or PV stenosis when using higher density of RF applications. That may be influenced by fact that during all periods, non-surround flow catheter was used.

The increasing use of ICE during our procedures also might have increased our efficacy. This is the only currently available imaging technique which can be used in real time during ablation. Combination of ICE images and CARTO map created using ablation catheter or circular navigating catheter probably improves creation of real LA map and delineating PV ostia. It also helps to avoid RF energy delivery inside PV and is useful in early detection of such complications as steam pops or pericardial effusion [7].

The changes in the $\mathrm{CB}$ technique during the analyzed period were not so pronounced as in the RF technology. The biggest change was the introduction of new generation of cryoballoons which enabled more effective tissue cooling around PV ostia [10]. Another change was the assessment of
Fig. 1 Overall efficacy (\%) in analyzed time-periods. Period I vs period II and vs period III, $p<0.05$

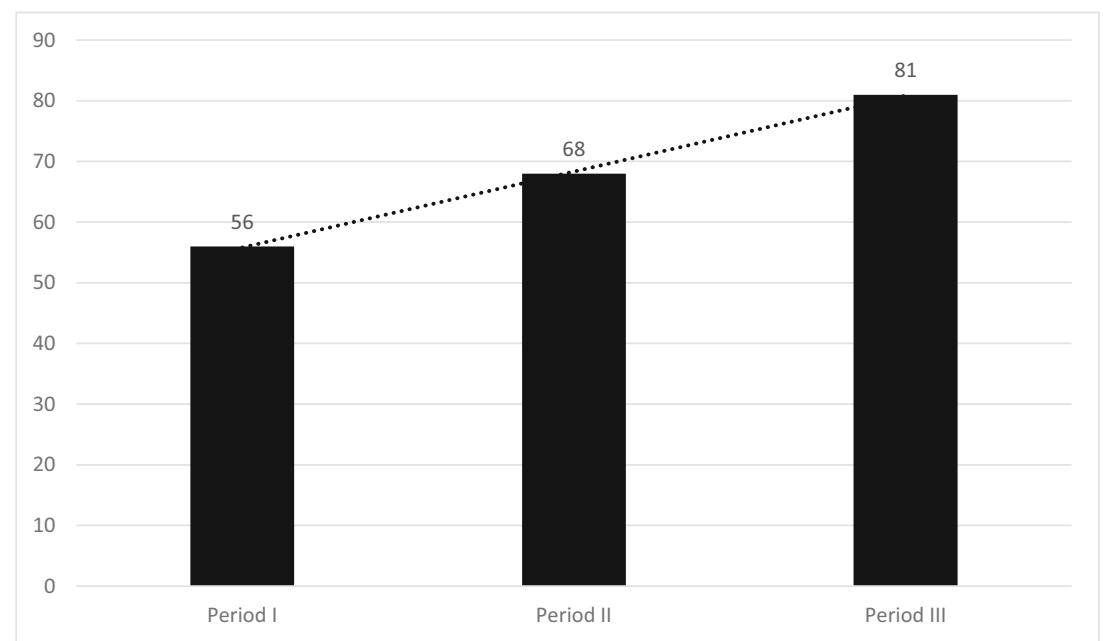


Fig. 2 Comparison of RF vs CRYO efficacy $(\%)$ in analyzed time-periods. For RF: Period I vs period II and vs period III, $p<0.01$; for $\mathrm{CB}$, NS

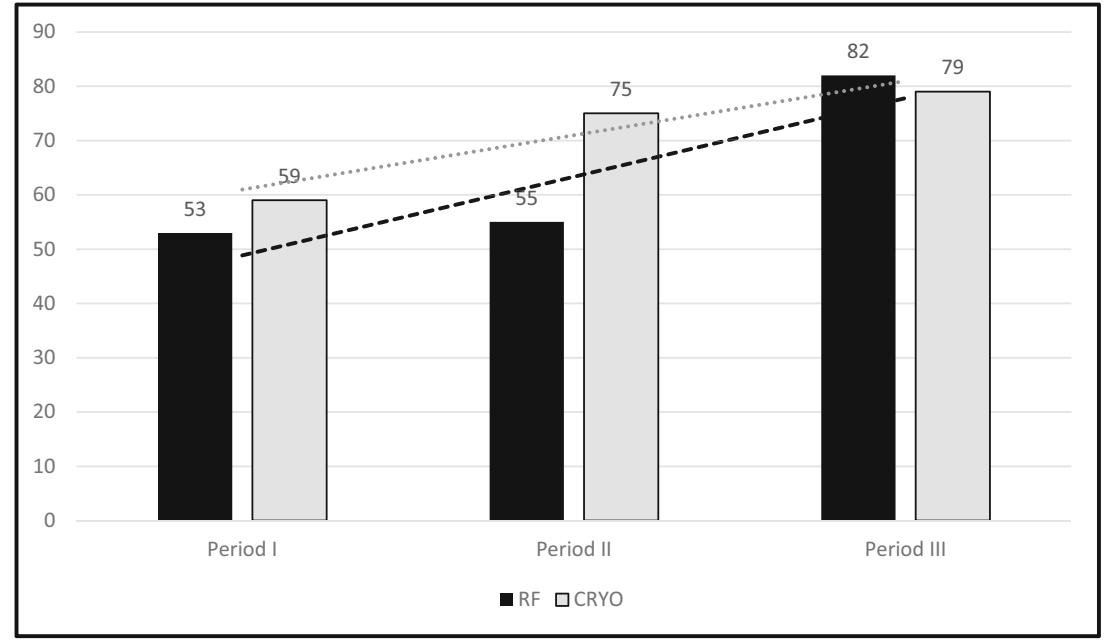

lesion quality by introducing time to PVI and thawing time as important parameters for efficacy assessment. Also, duration of cryoapplications started to be more individualized, based on these parameters as well maximal temperature achieved and quality of PV occlusion [11].

\subsection{Learning curve and operator's experience}

Learning curve is important in any procedure. According to literature, learning curve in RF point-by-point technique is quite long and may require 100 procedures to perform as the first operator to obtain high skills [12]. This probably also influenced the results in our patients and resulted in higher efficacy in the last analyzed period. Between 2012 and 2019, five operators performed over $700 \mathrm{AF}$ ablations in our institution and, in addition, over 300 procedures in five other institutions, where our team also works, which gives a total > 1000 AF ablations.

Learning curve in $\mathrm{CB}$ has been reported to be much shorter and only 20-30 procedures are enough to effectively and safely perform $\mathrm{CB} A F$ ablation procedures in patients with normal anatomy [13]. Some have even reported that the results may be similar in experienced and inexperienced centers [14]. However, there may be a significant difference in performance between an operator who is already experienced in RF AF ablation and an operator who just starts AF ablation procedures. Such anatomical issues as left PV common trunk, very small LA, additional PV, or very oval PV orifice may require more skilled operators [15]. Also, ICE images may help in proper balloon positioning in difficult cases [16].

\subsection{Changes in patients' characteristics and procedural parameters over time}

We did not observe any significant difference in patients' demographic or clinical characteristics during analyzed periods. In spite of increasing knowledge that obesity and overweight significantly reduce efficacy of AF ablation [17], we did not manage to correct this parameter in our patients who had mean BMI between 28 and 31. This shows that more effort is required for risk factor modification, perhaps by especially dedicated teams [17].

Table 2 Comparison of procedural details between three analyzed periods - RF

\begin{tabular}{|c|c|c|c|c|c|c|}
\hline Parameter & Period I & Period II & Period III & P I vs II & I vs III & II vs III \\
\hline Procedure duration (min) & $247 \pm 53$ & $254 \pm 64$ & $237 \pm 49$ & NS & NS & NS \\
\hline Fluoroscopy time (min) & $46.8 \pm 16.4$ & $22.1 \pm 16.5$ & $11.1 \pm 6.0$ & $<0.05$ & $<0.05$ & $<0.05$ \\
\hline \multicolumn{7}{|l|}{ RF details: } \\
\hline $\mathrm{RF}$ duration (sec) & $3735 \pm 1316$ & $2712 \pm 1106$ & $3381 \pm 881$ & $<0.05$ & NS & $<0.05$ \\
\hline Ablation count (number of RF applications) & $103 \pm 46$ & $49 \pm 19$ & $121 \pm 33$ & $<0.05$ & NS & $<0.05$ \\
\hline Number of isolated PV & $3.4 \pm 1.1$ & $3.9 \pm 0.4$ & 4 & $<0.05$ & $<0.05$ & NS \\
\hline \multicolumn{7}{|l|}{ ICE usage: } \\
\hline For TP only & $30(61 \%)$ & $26(90 \%)$ & $40(89 \%)$ & 0.007 & 0.002 & NS \\
\hline For TP and guiding procedure & 0 & $13(45 \%)$ & $40(89 \%)$ & 0.0001 & 0.0001 & 0.0001 \\
\hline
\end{tabular}

$R F$, radiofrequency; $P V$, pulmonary veins; $I C E$, intracardiac echocardiography; $T P$, transseptal puncture 
Table 3 Comparison of procedural details between three analyzed periods - CB

\begin{tabular}{|c|c|c|c|c|c|c|}
\hline Parameter & Period I & Period II & Period III & P I vs II & I vs III & II vs III \\
\hline Procedure duration (min) & $173 \pm 43$ & $144 \pm 48$ & $121 \pm 18$ & $<0.05$ & $<0.05$ & NS \\
\hline Fluoroscopy time (min) & $44.8 \pm 31.2$ & $25.3 \pm 14.4$ & $15.9 \pm 6.1$ & $<0.05$ & $<0.05$ & $<0.05$ \\
\hline Number of isolated PV & $3.6 \pm 0.9$ & 4 & 4 & $<0.05$ & $<0.05$ & NS \\
\hline \multicolumn{7}{|l|}{ Nadir temperature $\left({ }^{\circ} \mathrm{C}\right)$ : } \\
\hline LSPV & $-48 \pm 7$ & $-45 \pm 6$ & $-44 \pm 3$ & $<0.05$ & NS & NS \\
\hline LIPV & $-48 \pm 9$ & $-42 \pm 5$ & $-40 \pm 5$ & $<0.05$ & $<0.05$ & NS \\
\hline RSPV & $-47 \pm 8$ & $-48 \pm 5$ & $-49 \pm 5$ & NS & NS & NS \\
\hline RIPV & $-42 \pm 5$ & $-44 \pm 5$ & $-43 \pm 5$ & NS & NS & NS \\
\hline Total number of freezes & $9.3 \pm 2.4$ & $5.9 \pm 1.8$ & $5.5 \pm 0.8$ & $<0.05$ & $<0.00001$ & NS \\
\hline LSPV & $2.7 \pm 0.8$ & $1.4 \pm 0.6$ & $1.4 \pm 0.6$ & $<0.00001$ & $<0.00001$ & NS \\
\hline LIPV & $2.6 \pm 1.0$ & $1.7 \pm 1.0$ & $1.3 \pm 0.7$ & $<0.00001$ & 0.00002 & NS \\
\hline RIPV & $2.0 \pm 1.1$ & $1.4 \pm 0.7$ & $1.4 \pm 0.7$ & 0.013 & 0.021 & NS \\
\hline RSPV & $2.2 \pm 0.9$ & $1.5 \pm 0.7$ & $1.2 \pm 0.4$ & 0.0009 & $<0.00001$ & 0.02 \\
\hline Total freezing time sec) & $2535 \pm 862$ & $1284 \pm 329$ & $1127 \pm 191$ & $<0.05$ & $<0.05$ & NS \\
\hline LSPV & $719 \pm 272$ & $315 \pm 137$ & $262 \pm 84$ & $<0.00001$ & $<0.00001$ & 0.043 \\
\hline LIPV & $705 \pm 323$ & $368 \pm 174$ & $280 \pm 171$ & $<0.00001$ & $<0.00001$ & 0.046 \\
\hline RIPV & $538 \pm 335$ & $303 \pm 136$ & $288 \pm 160$ & $<0.0007$ & $<0.0007$ & NS \\
\hline RSPV & $596 \pm 301$ & $304 \pm 153$ & $223 \pm 90$ & $<0.00001$ & $<0.00001$ & 0.005 \\
\hline \multicolumn{7}{|l|}{ ICE usage: } \\
\hline For TP only & $17(53 \%)$ & $21(38 \%)$ & $19(79 \%)$ & NS & NS & 0.0008 \\
\hline For TP and guiding procedure & 0 & $5(9 \%)$ & $19(79 \%)$ & NS & $<0.00001$ & $<0.0001$ \\
\hline
\end{tabular}

$L S P V$, left superior PV; $L I P V$, left inferior PV; RIPV, right inferior PV; RSPV, right superior PV; rest of abbreviations, as in Table 2

Table 4 Complications

\begin{tabular}{|c|c|c|c|c|c|}
\hline Complications & Total $n=21(9 \%)$ & Period I $n=8$ & Period II $n=7$ & Period III $n=6$ & $\mathrm{RF} / \mathrm{CB}$ \\
\hline \multicolumn{6}{|l|}{ Major: } \\
\hline Cardiac tamponade & 1 & 0 & 0 & 1 & $0 / 1$ \\
\hline Significant peri-procedural pericardial effusion & 2 & 2 & 0 & 0 & $2 / 0$ \\
\hline Stroke & 1 & 0 & 0 & 1 & $1 / 0$ \\
\hline Transient ischemic attack & 1 & 1 & 0 & 0 & $1 / 0$ \\
\hline Right coronary artery air embolism following TP & 1 & 1 & 0 & 0 & $0 / 1$ \\
\hline Atrio-esophageal fistula & 0 & 0 & 0 & 0 & 0 \\
\hline Death & 0 & 0 & 0 & 0 & 0 \\
\hline Chronic pericarditis with large amount of pericardial fluid & 1 & 0 & 0 & 1 & $1 / 0$ \\
\hline Symptomatic PV stenosis & 1 & 0 & 1 & 0 & $1 / 0$ \\
\hline Persistent phrenic nerve paralysis & 0 & 0 & 0 & 0 & 0 \\
\hline Pulmonary segmental atelectasis & 1 & 1 & 0 & 0 & $0 / 1$ \\
\hline Major subtotal & 9 & 5 & 1 & 3 & $6 / 3$ \\
\hline Local (before/after introduction of USG-guided VA) & $12(7 / 5)$ & 3 & 6 & 3 & $8 / 4$ \\
\hline \multicolumn{6}{|l|}{ Including: } \\
\hline Arterio-venous fistula & $6(3 / 3)$ & 1 & 3 & 2 & $4 / 2$ \\
\hline Femoral artery pseudoaneurysm & $2(2 / 0)$ & 1 & 1 & 0 & $2 / 0$ \\
\hline $\begin{array}{l}\text { Significant hematoma (requiring blood } \\
\text { transfusion or prolonged hospitalization) }\end{array}$ & $4(2 / 2)$ & 1 & 2 & 1 & $2 / 2$ \\
\hline
\end{tabular}

$V A$, vascular access; rest of abbreviations, Table 2 


\subsection{Changes in procedural parameters}

Total duration of procedure reduced significantly in the $\mathrm{CB}$ group whereas it stayed constant in the RF group. The former result is expected and in line with data reported in literature $[10,13,14]$ whereas no change in duration of $\mathrm{RF}$ ablation is unexpected and may be due to more detailed and meticulous delivery of lesions now than in the past in our laboratory. The overall time of approximately $4 \mathrm{~h}$ is long; however, it includes LA appendage assessment using ICE and approximately 20 min waiting time for PV reconnection, testing for PV conduction and searching for non-PV triggers.

The decrease in fluoroscopy time using both RF and $\mathrm{CB}$ is expected, in line with literature [3,10,13,14], and reflects growing experience as well as advent of ICE, especially during RF ablation. After creating CARTO map, fluoroscopy is rarely used and almost all catheter movements and RF applications are guided by electro-anatomical map and ICE imaging. Also, an increase in the number of isolated PV which reached all $4 \mathrm{PV}$ using either technique is in line with reported data $[13,14]$. The reduction in fluoroscopy time in the CB arm between periods II and III was probably mainly due to the change of protocol from two freezes per vein to one freeze per vein. Further reduction in fluoroscopy time in the $C B$ group between period II and III was mainly due to increasing overall experience, efforts to reduce fluoroscopic imaging time after contrast injection and, most importantly, expanding usage of ICE during the procedure (see Table 3) which is an excellent tool for confirming PV occlusion (color Doppler).

Differences in total RF duration and ablation count depict changes in the quality of RF lesions. We started with numerous lesions, however, with some being of poor quality (period I), then continued with lower number of lesions with better but not optimal quality (period II) and observed renewed increase in the RF application number and duration (period III) as a result of the CLOSE protocol introduction. In periods I and II, dragging technique was allowed which also might have influenced the lesion count-and resulted in less ablation counts with longer duration per application. In the last period (period III), we observed renewed increase in the $\mathrm{RF}$ application number and duration as a result of the CLOSE protocol introduction.

Lastly, small differences in nadir temperature achieved during $\mathrm{CB}$ in left PV may depict our growing awareness concerning possible PV stenosis and collateral damage when too low temperatures are achieved [18].

Complications The frequency of complications was comparable to other real world reports $[4,6]$. We believe that a low rate of cardiac tamponade in our study is in part associated with the ICE use. Two neurological complications occurred in patients with many risk factors $\left(\mathrm{CHA}_{2} \mathrm{DS}_{2}\right.$ Vasc $\left.=5\right)$ or sub-optimal $\mathrm{ACT}$ during initial part of the procedure (maximal ACT of only $275 \mathrm{~s}$ was achieved). The rate of local complications was similar before and after introduction of USG-guided approach which shows that there is a room for improvement in our EP laboratory because data from literature suggest reduction of local complications when USG is used [19].

Limitations This is a single-center experience with relatively low number of patients, however, with consistent follow-up scheme, completed by $100 \%$ of patients. We did not use implantable cardiac monitors to detect AF recurrences; thus, asymptomatic PAF may be under-detected. However, frequent clinical assessment and repeated 7-day Holter ECG recordings seem the most frequently used tools in other studies and in everyday clinical practice. A significant proportion of patients were on antiarrhythmic drugs during follow-up; however, the European registry also showed that a high proportion $(46 \%)$ of patients remained on antiarrhythmic medication following AF ablation [4] so this depicts real-life situation. Follow-up duration was only 1 year-longer follow-up might have resulted in different results. There were differences in the proportion of RF vs CB usage between period II vs periods I and III which might have influenced the results. Extra-PV RF applications were performed in 4 patients from the RF group and one in the $\mathrm{CB}$ group. However, these numbers were really low (4 vs 1) and should have not influenced significantly the results.

\section{Conclusions}

This real-life observational study from a medium volume EP center confirms results from high-volume very experienced centers on the progress in efficacy and safety of AF ablation, especially using RF energy, observed in recent years.

Open Access This article is licensed under a Creative Commons Attribution 4.0 International License, which permits use, sharing, adaptation, distribution and reproduction in any medium or format, as long as you give appropriate credit to the original author(s) and the source, provide a link to the Creative Commons licence, and indicate if changes were made. The images or other third party material in this article are included in the article's Creative Commons licence, unless indicated otherwise in a credit line to the material. If material is not included in the article's Creative Commons licence and your intended use is not permitted by statutory regulation or exceeds the permitted use, you will need to obtain permission directly from the copyright holder. To view a copy of this licence, visit http://creativecommons.org/licenses/by/4.0/.

\section{References}

1. Haïssaguerre M, Jaïs P, Shah DC, Takahashi A, Hocini M, Quiniou $\mathrm{G}$, et al. Spontaneous initiation of atrial fibrillation by ectopic beats 
originating in the pulmonary veins. N Engl J Med. 1998;339:65966.

2. Taghji P, El Haddad M, Phlips T, Wolf M, Knecht S, Vandekerckhove Y, et al. Evaluation of a strategy aiming to enclose the pulmonary veins with contiguous and optimized radiofrequency lesions in paroxysmal atrial fibrillation: a pilot study. JACC Clin Electrophysiol. 2018;4:99-108.

3. Berte B, Hilfiker G, Moccetti F, Schefer T, Weberndörfer V, Cuculi $\mathrm{F}$, et al. Pulmonary vein isolation using ablation index vs. CLOSE protocol with a surround flow ablation catheter. Europace. 2020;22: 84-9. https://doi.org/10.1093/europace/euz244.

4. Arbelo E, Brugada J, Blomström-Lundqvist C, Laroche C, Kautzner J, Pokushalov E, et al. Contemporary management of patients undergoing atrial fibrillation ablation: in-hospital and 1year follow-up findings from the ESC-EHRA atrial fibrillation ablation long-term registry. Eur Heart J. 2017;38:1303-16. https:// doi.org/10.1093/eurheartj/ehw564.

5. Neyt M, Van Brabandt H, Devos C. The cost-utility of catheter ablation of atrial fibrillation: a systematic review and critical appraisal of economic evaluations. BMC Cardiovase Disord. 2013;13:78. https://doi.org/10.1186/1471-2261-13-78.

6. Cheng EP, Liu CF, Yeo I, Markowitz SM, Thomas G, Ip JE, et al. Risk of mortality following catheter ablation of atrial fibrillation. J Am Coll Cardiol. 2019;74(18):2254-64. https://doi.org/10.1016/j. jacc.2019.08.1036.

7. $2017 \mathrm{HRS} / \mathrm{EHRA} / \mathrm{ECAS} / \mathrm{APHRS} / \mathrm{SOLAECE}$ expert consensus statement on catheter and surgical ablation of atrial fibrillation: executive summary. J Arrhythm. 2017;33:369-409. https://doi. org/10.1016/j.joa.2017.08.001.

8. Pilichowska-Paszkiet E, Baran J, Sygitowicz G, Sikorska A, Stec S, Kułakowski P, et al. Noninvasive assessment of left atrial fibrosis. Correlation between echocardiography, biomarkers, and electroanatomical mapping. Echocardiography. 2018;35:1326-34.

9. Sikorska A, Pilichowska-Paszkiet E, Zuk A, Piotrowski R, Kryński $\mathrm{T}$, Baran J, et al. Acceleration of sinus rhythm following ablation for atrial fibrillation: a simple parameter predicting ablation efficacy. Kardiol Pol. 2019;77:960-5.

10. Straube F, Dorwarth U, Schmidt M, Wankerl M, Ebersberger U, Hoffman E. Compariosn of the first and second cryoballon: highvolume single-venter safety and efficacy analysis. Circ Arrhythm Electrophysiol. 2014;7:293-9.

11. Miyazaki S, Kajiyama T, Watanabe T, Nakamura H, Hachiya H, Tada H, et al. Predictors of durable pulmonary vein isolation after second-generation cryoballoon ablation with a single short freeze strategy - different criteria for the best freeze of the 4 individual PVs. Int J Cardiol. 2020;301:96-102. https://doi.org/10.1016/j. ijcard.2019.11.089.

12. Sairaku A, Nakano Y, Oda N, Makita Y, Kajihara K, Tokuyama T, et al. Learning curve for ablation of atrial fibrillation in mediumvolume centers. J Cardiol. 2011;57:263-8.

13. Velagić V, de Asmundis C, Mugnai G, Hünük B, Hacioğlu E, Ströker E, et al. Learning curve using the second-generation cryoballoon ablation. J Cardiovasc Med (Hagerstown). 2017;18: 518-27.

14. Landolina M, Arena G, Iacopino S, et al. Center experience does not influence long-term outcome and peri-procedural complications after cryoballoon ablation of paroxysmal atrial fibrillation: data on 860 patients from the real-world multicenter observational project. Int J Cardiol. 2018;272:130-6.

15. Baran J, Piotrowski R, Sikorska A, Kowalik I, Kryński T, Stec S, et al. Impact of pulmonary vein ostia anatomy on efficacy of cryoballoon ablation for atrial fibrillation. Heart Beat J. 2016;1: $65-70$.

16. Rubesch-Kütemeyer V, Molatta S, Vogt J, Gutleben KJ, Horstkotte D, Nölker G, et al. Reduction of radiation exposure in cryoballoon ablation procedures: a single-centre study applying intracardiac echocardiography and other radioprotective measures. Europace. 2017;19(6):947-53. https://doi.org/10.1093/europace/euw139.

17. Middeldorp ME, Pathak RK, Meredith M, Mehta AB, Elliott AD, Mahajan R, et al. PREVEntion and regReSsive Effect of weightloss and risk factor modification on atrial fibrillation: the REVERSE-AF study. Europace. 2018;20:1929-35. https://doi. org/10.1093/europace/euy117.

18. Baran J, Lewandowski P, Smarż K, Sikorska A, Zaborska B, Kułakowski P. Acute hemodynamic and tissue effects of cryoballoon ablation on pulmonary vessels: the IVUS-Cryo study. J Am Heart Assoc. 2017;6(6):e005988. https://doi.org/10.1161/ JAHA.117.005988.

19. Futyma P, Ciąpała K, Sander J, Głuszczyk R, Futyma M, Kułakowski P. Ultrasound-guided venous access facilitated by the Valsalva maneuver during invasive electrophysiological procedures. Kardiol Pol. 2020;78(3):235-9. https://doi.org/10.33963/ KP.15188.

Publisher's note Springer Nature remains neutral with regard to jurisdictional claims in published maps and institutional affiliations. 